\title{
Future Prospective of Regenerative Therapy of the Central Nervous System
}

\section{Athanasios K Petridis*}

Department of Neurosurgery, University of Schleswig Holstein, Germany

In the last decades there have been huge leaps in the field of regenerative therapeutic approaches for diseases of the central nervous system (CNS). Stem cell transplantation and identification of molecules crucial for regenerative processes took the main part of scientific projects. By understanding neuronal development and mimicking developmental steps, i.e. trying to induce expression of molecules expressed during neuronal development when injury to the central nervous system occurs, could lead to the effect regeneration scientist all over the world are aiming for.

Polysialic acid, a polysaccharide with a high affinity to bind water molecules, (PSA) as an example, and as the molecule of my personal focus, is over-expressed during neuronal development. After maturation of the CNS, expression of PSA is down regulated and the polysaccharide is expressed only in areas were neuronal stem cells are generated like the subventricular zone (SVZ). The function of PSA is mainly to enable migration of neuronal stem cells from the SVZ to the olfactory bulb were neuronal cells are substituted throughout life. Another function of PSA is to delay cell maturation.

Apart from these effects, it has been observed that PSA is temporary re-expressed in glial cells after injury in the CNS. These observation together with the known function of PSA to enable cells as well as neuronal processes to move ( or grow in case of neuronal processes) is used in our experimental studies at the Memorial Sloan Kettering Cancer Center from 2002-2005. PSA was over expressed specifically in glial cells after CNS injury in the brain and spinal cord. This PSA rich environment allowed stem cells to move from the CNS to brain areas which have been injured. In spinal cord lesions the PSA rich gliotic scar enabled neuronal processes to grow through the gliotic scar and pass the scar. A huge wall in regeneration of the CNS, namely the gliotic scar was successfully overpassed [1].

Another interesting observation was that PSA is used also by tumor cells. In highly metastasizing tumors and in tumors which spread fast to their environment, like glioblastomas, PSA is highly expressed. Tumor cells use the effect of PSA to keep them away from neighbour cells. Therefore the cells can stay isolated without contact to other cells and avoiding cell-cell-interactions which could even lead to a re differentiation of tumor cells. Tumor cells can keep their antisocial, anarchistic character through the expression of PSA. Studies have shown that cleavage of PSA by its specific endoneuraminidase, endo $\mathrm{N}$ can lead to re differentiation of neuroblastoma cells and to a decrease of their migratory potential $[2,3]$.

Studies are now planned to explore in which degree the PSA expression enhances the malignant potential of tumors and how the endo $\mathrm{N}$ cleavage could delay tumor growth. The results will be very interesting since a future perspective in treatment of PSA rich tumors with endo $\mathrm{N}$ could become reality.

\section{References}

1. El Maarouf A, Petridis AK, Rutishauser U (2006) Use of polysialic acid in repair of the central nervous system. Proc Natl Acad Sci U S A 103: 16989 16994.

2. Petridis AK, El-Maarouf A, Rutishauser U (2004) Polysialic acid regulates cell contact-dependent neuronal differentiation of progenitor cells from the subventricular zone. Dev Dyn 230: 675-684.

3. Petridis AK, Wedderkopp H, Hugo HH, Maximilian Mehdorn H (2009) Polysialic acid overexpression in malignant astrocytomas. Acta Neurochir (Wien) 151: 601-603.
${ }^{*}$ Corresponding author: Athanasios K. Petridis, Associate Professor, Department of Neurosurgery, University of Schleswig Holstein, Germany, E-mail: opticdisc@aol.com

Received December 06, 2012; Accepted December 06, 2012; Published December 08, 2012

Citation: Petridis AK (2013) Future Prospective of Regenerative Therapy of the Central Nervous System. J Neurol Disord 1:e101. doi:10.4172/2329$6895.1000 \mathrm{e} 101$

Copyright: (C) 2013 Petridis AK. This is an open-access article distributed under the terms of the Creative Commons Attribution License, which permits unrestricted use, distribution, and reproduction in any medium, provided the original author and source are credited. 